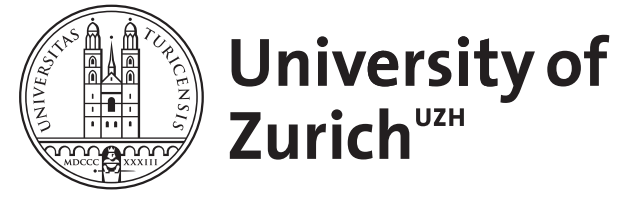

\title{
Chimpanzee termite fishing etiquette
}

Koops, Kathelijne

\begin{abstract}
Human culture is unique. Or is it? A new study reveals unexpected cultural diversity in the fine-grained details of chimpanzee termite fishing behaviour. These novel findings shed light on the richness of chimpanzee cultural diversity and reveal a narrower gap between the cultures of humans and other apes.
\end{abstract}

DOI: https://doi.org/10.1038/s41562-020-0895-9

Posted at the Zurich Open Repository and Archive, University of Zurich ZORA URL: https://doi.org/10.5167/uzh-198827

Journal Article

Accepted Version

Originally published at:

Koops, Kathelijne (2020). Chimpanzee termite fishing etiquette. Nature Human Behaviour, 4(9):887-888. DOI: https://doi.org/10.1038/s41562-020-0895-9 


\section{PRIMATOLOGY}

\section{Chimpanzee termite fishing etiquette}

3

$4 \quad$ Kathelijne Koops

Department of Archaeology, University of Cambridge, Cambridge, UK

Corresponding author: Kathelijne Koops (kk370@cam.ac.uk)

Human culture is unique. Or is it? A new study reveals unexpected cultural diversity in the fine-grained details of chimpanzee termite fishing behaviour. These novel findings shed light on the richness of chimpanzee cultural diversity and reveal a narrower gap between the cultures of humans and other apes.

Complex culture has long been considered a uniquely human trait. Our cultural norms influence all facets of daily life, ranging from the way we eat to the way we socialize. But, is this actually unique to humans? Ever since Jane Goodall first described wild chimpanzees using blades of grass to fish for termites ${ }^{1}$, the view of humans as the only species to have (material) culture has been in question. In the pivotal paper by Andrew Whiten and colleagues, chimpanzee culture was first put on the map ${ }^{2}$. In that paper, scientists compared many different behaviours of wild chimpanzees across seven study groups and designated behaviours as 'cultural' when genetic and ecological explanations for behavioural differences could be excluded ${ }^{2}$. The use of tools by chimpanzees was found to be part of what we now call 'chimpanzee culture'. However, if chimpanzees have culture, and if that culture varies among populations just as it does between human societies, is there anything in human culture that still marks it as different from non-human animal cultures? Surely, humans are unique?

One of the main proposed differences between human culture and the cultures of other species is the level of cultural complexity. Human culture is considered cumulative, which means that as skills and technologies accumulate over generations they become more complex than what one individual could invent alone ${ }^{3}$. Cumulative culture is only possible if the mechanism of cultural transmission is sufficiently reliable. Think of imitating the actions of a skilled person, or of being actively taught how to do something. Such high-fidelity transmission mechanisms (imitation and teaching) are considered unique to humans by some scientists ${ }^{3}$. Chimpanzee cultural behaviours, however, are proposed to be simple enough that they can be independently invented by each individual chimpanzee without the use of more reliable learning mechanisms. The jury is still out. 
The big problem in assessing the differences between human and chimpanzee cultural complexity is the number of populations included in these comparisons. Ethnographic studies of human culture include hundreds of societies often studied in great detail, whereas comparative studies of chimpanzee culture are limited to about a dozen populations. Hence, chimpanzee cultural complexity may be considerably underestimated due to such limited sampling. A new study by Christophe Boesch and colleagues published in Nature Human Behaviour sets the record straight ${ }^{4}$. The authors present findings from an unparalleled effort sampling 39 chimpanzee populations across the African continent, part of a project called the Pan African Programme. This research programme has applied state of the art camera trapping to allow non-invasive observations of chimpanzee communities not habituated to the presence of researchers. Boesch and colleagues focus specifically on the first chimpanzee cultural behaviour discovered and studied extensively since: termite fishing.

Since the early descriptions at Gombe (Tanzania), researchers have recorded termite fishing in seven other chimpanzee populations. However, direct observations of termite fishing techniques were restricted to just two study sites. Boesch and colleagues now provide detailed records of termite fishing for nine additional study sites, bringing the total to 11 . This unprecedented sampling effort reveals a staggering total of 38 different technical elements in termite fishing (up from the previous report of two). Importantly, 30 of these elements were not constrained by site-specific ecological challenges, such as termite mound structure, and could thus be investigated as termite fishing culture. Not only did the frequency of occurrence of different technical elements differ between chimpanzee communities, but the researchers also discovered that individuals living in the same community have similar termite fishing techniques and show community-specific combinations of technical elements. For example, Korup chimpanzees in Cameroon fish for termites whilst leaning on an elbow, which they combine with other community-specific elements such as shaking the tool with their lips whilst fishing. Chimpanzees at Wonga Wongue in Gabon, on the other hand, can be recognized by their unique style of laying on their side whilst termite fishing. In sum, the authors uncovered both exceptional cultural diversity in termite fishing techniques, as well as community-specific termite fishing 'etiquettes'.

These findings have a number of important implications for our perception of human cultural uniqueness. First, the community-specific combinations of termite fishing behavioural elements (despite the availability of possible alternatives) strongly suggests that chimpanzees acquire these techniques via social learning mechanisms (which are supposedly unique to humans), like imitation or teaching, rather than by independent invention. In support of this, a recent study of termite-fishing acquisition by young chimpanzees at Goualougo (Republic of Congo) showed that 
mothers share their tools as a way of teaching their youngsters this complex tool use skill ${ }^{5}$. If chimpanzees are indeed capable of using these high-fidelity social learning mechanisms, cumulative culture may not be out of reach. Some would argue that the efficiency of termite fishing would have to be improved by combining technical elements in order for it to qualify as truly cumulative culture $^{3}$. Hence, future research will need to address the chronology of acquisition of the technical elements used in termite fishing, along with the learning mechanisms involved and the related fishing efficiencies.

The findings of Boesch and colleagues highlight the importance of including additional chimpanzee populations in comparative research on cultural diversity. With the inclusion of even more study sites in the future, the diversity of cultural traits will certainly continue to grow ${ }^{6}$. An interesting line of enquiry will be to examine how the different combinations of termite fishing elements cluster within communities. Are there differences in techniques between matrilines, similar to what has been reported for chimpanzee grooming styles ${ }^{7}$ (Fig. 1)? Another topic that requires further investigation is the link between termite behaviour and chimpanzee fishing technique. Previous research has found that the length of tools and the techniques used by chimpanzees to harvest army ants is partly influenced by the behaviour of the ant species preyed upon $^{8}$. Future work would do well to explore the interplay between ecology and culture ${ }^{9}$, in terms of termite prey species and fishing techniques. Each chimpanzee community adds a piece to the puzzle of the cultural richness of our ape cousins. However, only urgent efforts will ensure the protection of the highly endangered chimpanzee communities across Africa and maintain chimpanzee cultural diversity in the wild ${ }^{10}$.

\section{References}

1. Goodall, J. Nature 201, 1264-1266 (1964).

2. Whiten, A. et al. Nature 399, 682-685 (1999).

3. Tennie, C., Call, J. \& Tomasello, M. Phil. Trans. Roy. Soc. B. 364, 2405-2415 (2009).

4. Boesch, C. et al. Nature Human Behaviour. ... (2020).

5. Musgrave, S. et al. Proc. Natl. Acad. Sci. USA 117, 969-976 (2020).

6. Schuppli, C. \& van Schaik, C.P. Evol. Hum. Sci. 1: e2 (2019).

7. Wrangham, R.W. et al. Curr. Biol. 26, 3033-3037 (2016).

8. Schöning, C. et al. J. Hum. Evol. 55, 48-59 (2008).

9. Koops, K., Visalberghi, E. \& van Schaik, C.P. Biol. Let. 10, 20140508 (2014).

10. Kühl, H.S. et al. Science 363, 1453-1455 (2019). 


\section{Competing interests}

103 The author declares no competing interests.

104

105

106 Figure legend

107 Figure 1. High-arm grooming in the Kanyawara chimpanzees of the Kibale Forest, Uganda (C) 108 Kathelijne Koops). 
